\title{
Long-term preservation of antigenicity on tissue microarrays
}

\author{
Kyle A DiVito*, Lori A Charette*, David L Rimm and Robert L Camp \\ Department of Pathology, Yale University, New Haven, CT, USA
}

\begin{abstract}
Tissue microarrays have facilitated the evaluation of large cohort studies; however, there is little data on the best method for preserving sections once they are cut. We assessed three methods of storing precut breast cancer microarray slides: paraffin coating and storage in a nitrogen desiccator, either alone or in combination. We tested the durability of three antigens, cytokeratin, estrogen receptor, and Ki-67 on microarrays stored under these conditions for $\mathbf{3}$ months at room temperature. Staining was assessed with both manual scoring using traditional brown stain $(0-3+)$ as well as automated scoring using fluorescently stained sections. Staining intensity was compared to that obtained from freshly cut slides. Slides stored under ambient conditions (room temperature and air) for 3 months exhibited marked degradation of all target antigens, in some cases resulting in slides that were virtually unreadable. We found that combined paraffin coating and nitrogen storage resulted in the best preservation of antigenicity, with retention of $72-99 \%$ of the antigenicity of a freshly cut slide, depending upon the marker and detection system used. The use of either paraffin coating or nitrogen storage alone protected slides to a lesser degree.
\end{abstract}

Laboratory Investigation (2004) 84, 1071-1078, advance online publication, 14 June 2004; doi:10.1038/labinvest.3700131

Keywords: breast cancer; tissue microarray; antigen preservation; immunohistochemistry; tumor markers; biomarkers

Paraffin embedding of formalin-fixed tissue is a remarkably resilient method of specimen and antigen preservation. ${ }^{1,2}$ However, once samples from such blocks are cut into $5 \mu \mathrm{m}$ sections and deparaffinized, the tissue is rapidly exposed to ambient air and the potential effects of oxidation. Several studies have demonstrated the deleterious effects of storing precut tissue slides under ambient conditions; however, none have found a suitable method for storing slides which mitigates the loss of antigenicity. ${ }^{3-7}$ The lack of a suitable storage solution has a major impact on the viability of large cohort studies that rely on the collection and storage of slides for future batch processing. The advent of tissue microarrays as a tool for the study of immunohistochemical stains on large cohorts of tissue specimens arrayed in a single paraffin block has renewed interest in finding methods for cutslide preservation. ${ }^{7}$ To avoid section loss due to facing the block, serial sections of tissue microarrays are often cut at one time and stored as individual

Correspondence: Dr RL Camp, MD, PhD, Department of Pathology, Yale University, School of Medicine, 310 Cedar St, New Haven, CT 06520, USA.

E-mail: robert.camp@yale.edu

${ }^{*}$ Contributed equally to this manuscript.

Received 15 April 2004; revised and accepted 28 April 2004; published online 14 June 2004 slides. Therefore, maintaining antigenicity after sectioning is vital.

Toward the goal of developing a reliable method for the long-term storage of tissue microarray slides, we have employed two techniques: paraffin-coating of slides and storage in a nitrogen desiccation chamber. Our studies demonstrate that a combination of these two techniques can adequately protect tissue antigenicity for a period of up to 3 months.

\section{Materials and methods}

Tissue Microarrays

Breast arrays were obtained from the tissue microarray facility at Yale University Cancer Center, and contained 200 cases of primary carcinoma, half of which were node-negative, half node-positive. For Ki67 studies, a separate microarray consisting of 180 cores of 18 tumor types (including breast, colon, and melanoma) was used. Serial $5 \mu \mathrm{m}$ sections were cut and tape-transferred to adhesive slides according to standard protocols (Instrumedics, Inc., Hackensack, NJ, USA). After transfer, slides were washed in xylene and air-dried. When dry, some slides were coated by dipping them in melted paraffin. In some cases, paraffin was supplemented with 2,6-di-tertbutyl-4-methyl-phenol (BHT) or 2[3]-t-butyl-4- 
hydroxyanisole (BHA) (Sigma, St Louis, MO, USA). Slides were then stored at room temperature in ambient air or in a desiccating nitrogen chamber, maintained according to the manufacturers' specifications (Terra Universal, Anaheim, CA, USA). Slides were stored for 3 months, at which time the original paraffin microarray block was recut to produce 'freshly cut' control slides.

\section{Immunohistochemistry}

Slides were deparaffinized in two changes of xylene followed by two changes of absolute ethanol, and rehydrated in tap water. Antigen retrieval was performed by pressure cooking slides for $10 \mathrm{~min}$ in $6.5 \mathrm{mM}$ sodium citrate buffer ( $\mathrm{pH}$ 6.0). Slides were incubated in absolute methanol containing $0.6 \%$ hydrogen peroxide for $30 \mathrm{~min}$, to block endogenous peroxidases. Slides were again rinsed in tap water and blocked in Tris-buffered saline ( $\mathrm{pH}$ 8.0) containing $0.3 \%$ bovine serum albumin, for $30 \mathrm{~min}$. Slides for manual (visual) analysis were incubated for $1 \mathrm{~h}$ at room temperature with either mouse anti-ER antibody (DAKO Cytomation, Carpinteria, CA, USA) or mouse anti-Ki67 (BD Biosciences, San Jose, CA, USA). Slides were then incubated for $1 \mathrm{~h}$ with a horseradish peroxidase-conjugated secondary (Envision, DAKO Cytomation), and visualized using a 3, 3'-diaminobenzidine (DAB) chromogen. Staining for Ki-67 and ER was graded on a $0-3+$ scale. Slides for automated analysis were preincubated overnight at $4{ }^{\circ} \mathrm{C}$ with a rabbit anti-pan-cytokeratin (DAKO Cytomation) followed by the aforementioned anti-ER antibody and Envision secondary. For visualization, slides were then incubated for $1 \mathrm{~h}$ at room temperature with a fluorescent Alexa-488tagged goat anti-rabbit antibody (Molecular Bioprobes, Eugene, OR, USA) to visualize cytokeratin, and 4',6-diamidino-2-phenylindole, dihydrochloride (DAPI) to visualize nuclei. ER was visualized using a fluorescent chromagen, Cy5-tyramide (Perkin-Elmer Boston, MA, USA). Cy-5 (red) was used because its emission peak is well outside the greenorange spectrum of tissue autofluorescence.

\section{Automated Image Acquisition and Analysis}

Automated image acquisition and analysis using AQUA has been previously described. ${ }^{8}$ In brief, monochromatic, high-resolution $(1024 \times 1024$ pixel, $0.5 \mu \mathrm{m}$ resolution) images were obtained of each histospot. Areas of tumor were distinguished from stromal elements by creating a mask from the cytokeratin signal, and DAPI was used to identify nuclei. ER and cytokeratin signal from the membrane area of tumor cells was scored on a scale of 0-255, and expressed as signal intensity divided by the nuclear and membrane areas, respectively.

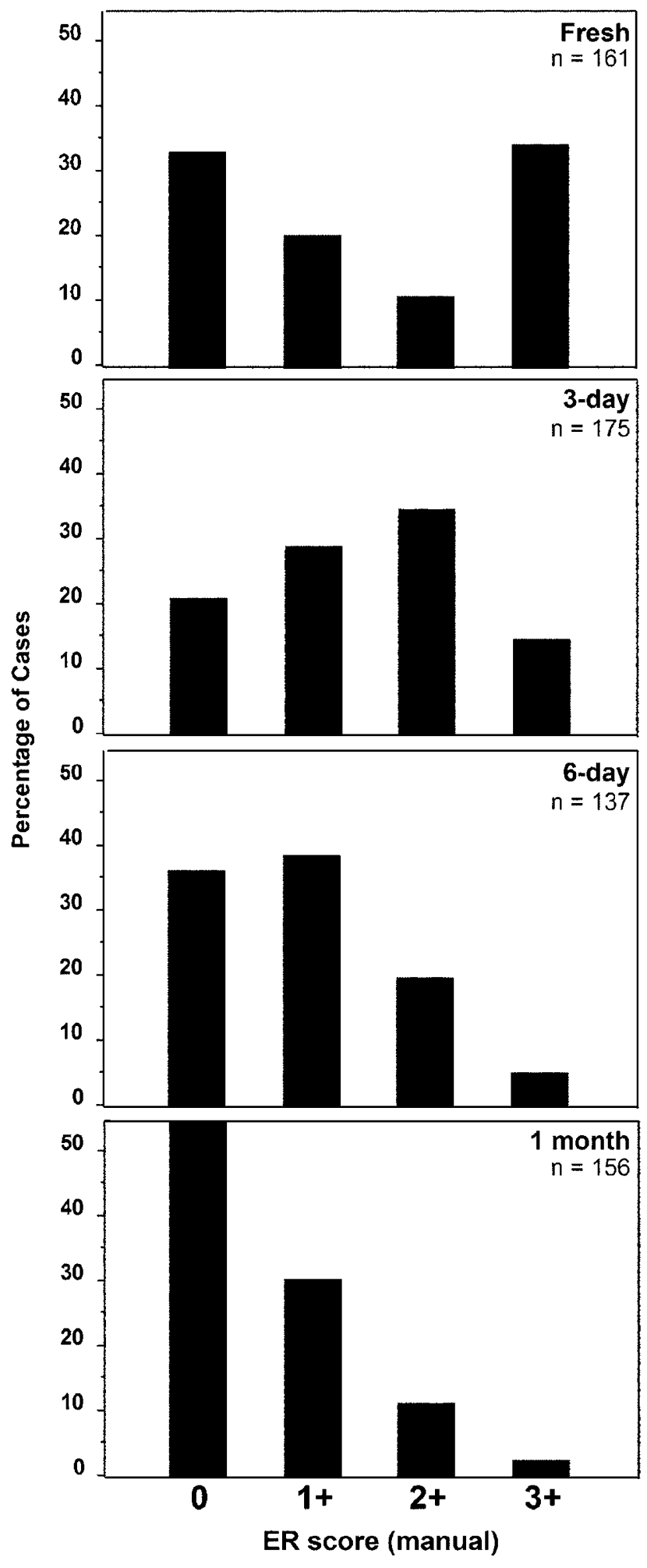

Figure 1 Slides stored under ambient conditions rapidly lose antigenicity for estrogen receptor. Tissue sections ( $5 \mu \mathrm{m})$ of a 200 case breast cancer microarray were cut and stained for ER after storage at room temperature in room air for 2-, 6-, or 30 days. Slides were scored manually on a four-point $(0-3+)$ scale. The percentage of cases scoring $0,1+, 2+$, or $3+$ on a freshly cut slide (top panel) are compared to scores from the stored slides (lower panels). 


\section{Results and discussion}

Precut Microarray Slides Stored under Ambient Conditions (Room Temperature and Air) Rapidly Lose Antigenicity

To determine the speed with which ER loses its antigenicity, we cut three, $5 \mu \mathrm{m}$ sections of our breast tissue microarray and stored them at room temperature, under room air for 2, 6, and 30 days. At each time point, an additional slide was freshly cut, and both slides were stained with ER and scored manually. The results of this staining are shown in Figures 1 and 2 and Table 1. This analysis shows that tissue microarrays stored in room air lose detectable antigenicity over the course of 1 month, with a statistically significant difference by 6 days.

\section{Combined Nitrogen Storage and Paraffin Coating Preserves Tissue Antigenicity}

We investigated two methods, storage in a nitrogen desiccator and paraffin coating, as potential methods

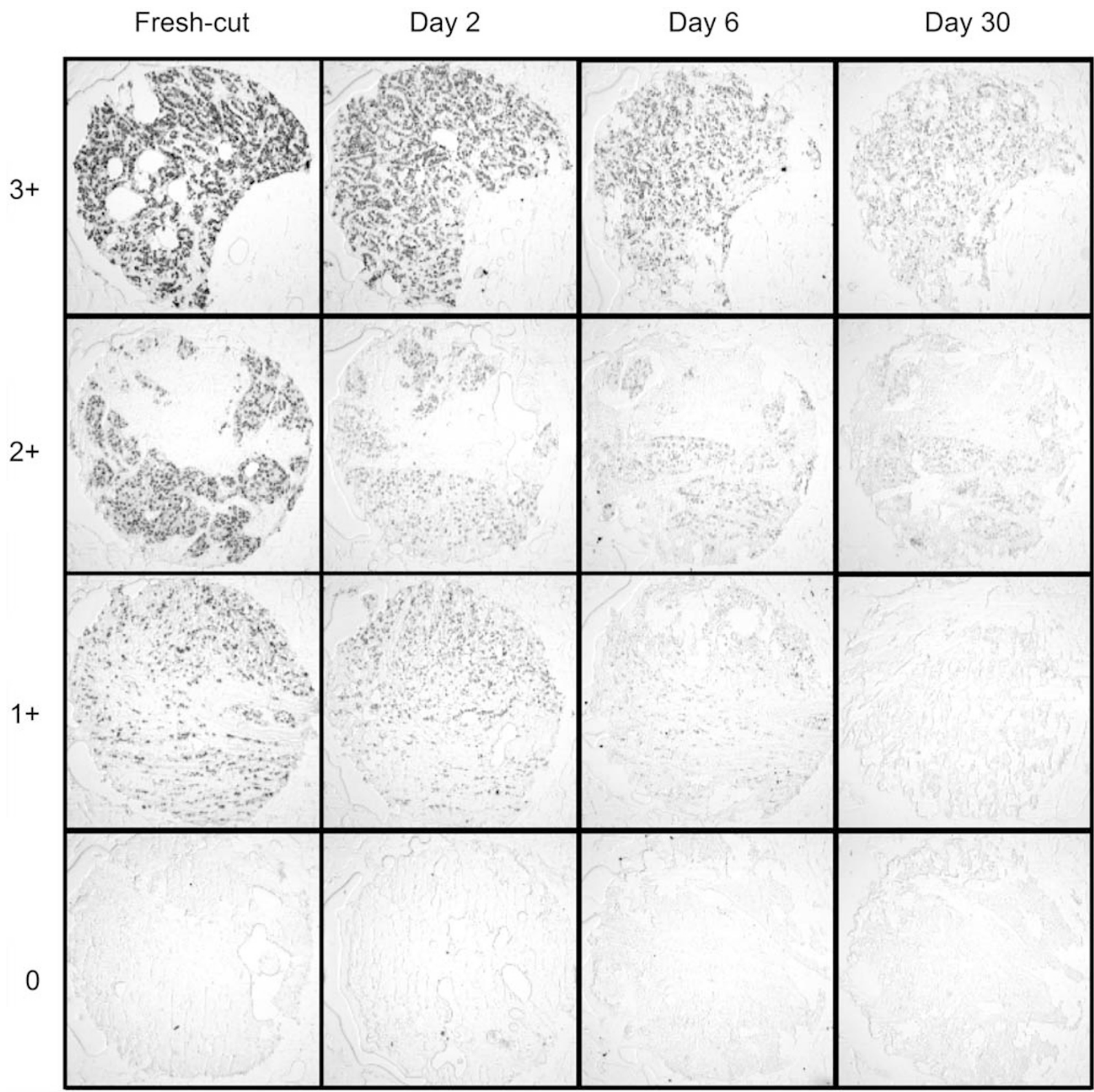

Figure 2 Estrogen receptor immunohistochemistry is abrogated by storage under ambient conditions. Representative, matched histospots $(0-3+)$ from freshly-cut slides as well as slides stored for 2-, 6-, or 30 days are shown. Slides were stained for ER and visualized with DAB. For clarity, histospots were not counterstained; however, each histospot presented is covered with greater than $50 \%$ tumor. Staining is localized to the tumor nuclei when assayed under high power. 
Table 1 Paired T-test of mean ER scores of slides stored under ambient conditions

\begin{tabular}{lrr}
\hline \multirow{2}{*}{ Time points } & \multicolumn{2}{c}{ Manual score } \\
\cline { 2 - 3 } & $\%$ Fresh & P-value \\
\hline Fresh & 100 & 0.1121 \\
2-day & 97 & $<0.0001$ \\
6-day & 63 & $<0.0001$ \\
30-day & 42 &
\end{tabular}

Table 2 Paired T-test of mean ER scores of stored slides stored for 3 months with nitrogen desiccation and/or paraffin coating

\begin{tabular}{lrrrrrr}
\hline \multirow{2}{*}{ Condition } & \multicolumn{2}{c}{ Manual score } & & \multicolumn{2}{c}{ Automated score } \\
\cline { 2 - 3 } & \% Fresh & P-value & & \% Fresh & P-value \\
& 100 & & & 100 & \\
Fresh & 77 & $<0.0001$ & & 43 & $<0.0001$ \\
Uncoated, room-air & 98 & 0.2266 & & 61 & $<0.0001$ \\
Uncoated, nitrogen & 90 & 0.3904 & & 82 & 0.0036 \\
Paraffin coated, room-air & 99 & 0.1598 & & 92 & 0.0070 \\
Paraffin coated, nitrogen & 99 & & & & \\
\hline
\end{tabular}

of preserving tissue antigenicity for the longer-term storage of cut slides. Our results on ER are presented in Figure 3 and Table 2. The results of ER immunohistochemistry were assayed both by manual scoring and automated (AQUA) analysis. As before, a substantial proportion of the ER staining was removed by storage under ambient conditions. Surprisingly, we noted less antigen loss at 3 months than in our original 1 month trial, by manual analysis (Tables 1 and 2). We attribute this to the uncontrolled nature of ambient storage conditions where humidity, in particular, may have preferentially influenced one trial more than the other. Both manual and automated analysis detected the loss of ER antigenicity; however, the magnitude of the loss was greater when assayed using automated analysis. This difference is principally due to the increased sensitivity of automated analysis for ER low positives, which under manual analysis were judged as $0 \mathrm{~s}$ and therefore had no ability to fall to a lower score. Excluding cases manually scored as 0 on the fresh-cut section, brings the retention detected by manual analysis closer to that detected by automated analysis (56 vs 43\%). Alone, both nitrogen storage and paraffin coating were protective; however, together they demonstrated superior ability to prevent antigen degradation. Although the antigen loss at 3 months under combined nitrogen storage and paraffin coating was statistically significant, it reflected at most only an $8 \%$ loss relative to a freshly cut slide.

We then determined if other antigens would exhibit similar signal loss, and whether this loss could be abrogated by nitrogen storage and/or paraffin coating. We assayed cytokeratin expression on our existing breast cancer array using automated analysis, and Ki-67 expression on a separate multitumor microarray using manual scoring. Despite its high level of expression in most breast carcinomas, cytokeratin proved to be dramatically sensitive to storage under ambient conditions, losing $88 \%$ of its signal by 3 months (Figure 4 , Table 3). As before, nitrogen storage and paraffin coating were partially protective, whereas combining the two techniques was almost fully protective, resulting in a nonsignificant loss of only $4 \%$. Manual analysis of Ki-67 demonstrated similar results; however, combined paraffin coating and nitrogen storage resulted in only a $72 \%$ retention of the antigenicity of a freshly cut slide (Figure 5, Table 4).

Finally, given the potential effects of oxidation, we attempted to augment the protective effects of paraffin coating by adding wax-soluble antioxidants (Figure 6 , Table 5 ). We added 1 and $10 \%(\mathrm{w} / \mathrm{v})$ of BHT or BHA to the paraffin used to coat tissue microarray slides and stored them for 5 months under nitrogen. Slides were stained for ER and analyzed using AQUA. Similar to our 3-month storage results, we found that nitrogen-stored paraffincoated slides retained $80 \%$ protective of the immunoreactivity of a freshly cut slide $(P<0.0001)$. Neither of the antioxidants at either concentration augmented this value; in fact, the addition of BHT or BHA consistently reduced the protective effects of paraffin alone $(P<0.0001)$. Given that we see no significant decrease in the immunoreactivity of slides stored for 5 vs 3 months (80 and $76 \%$ retention, respectively) and that the addition of antioxidants does not enhance the protective effects of paraffin alone, it is likely that there is a significant oxidative insult to the sections which occurs at the time of cutting. Consequently, paraffin-coating and nitrogen storage serve only to protect the slides from subsequent oxidation over time.

Prior studies have documented the loss of immunogenicity on precut slides over time,${ }^{3-6}$ including recently, tissue microarrays. ${ }^{7}$ Several of these studies assessed the benefit of storing slides at various temperatures, and in some cases coated with paraffin. In general, cold storage of slides $\left(4^{\circ} \mathrm{C}\right)$ was the optimal method of slide storage, but still resulted in significant losses of antigenicity. ${ }^{5,6}$ Paraffin coating was also found to be an ineffective method of antigen preservation on its own. ${ }^{5} \mathrm{Re}-$ cently, several researchers have reported on the benefits of new, non-formalin-based fixatives. These include HOPE fixative, ${ }^{9}$ zinc fixation. ${ }^{10,11}$ ethanol, ${ }^{12,13}$ and UMFIX. ${ }^{14}$ These fixatives show great promise in overcoming the deleterious effects of formalin-based fixation, and may also improve the durability of precut slides. However, they are not useful for retrospective studies on paraffinembedded archival tissues, the vast majority of which are formalin fixed. 

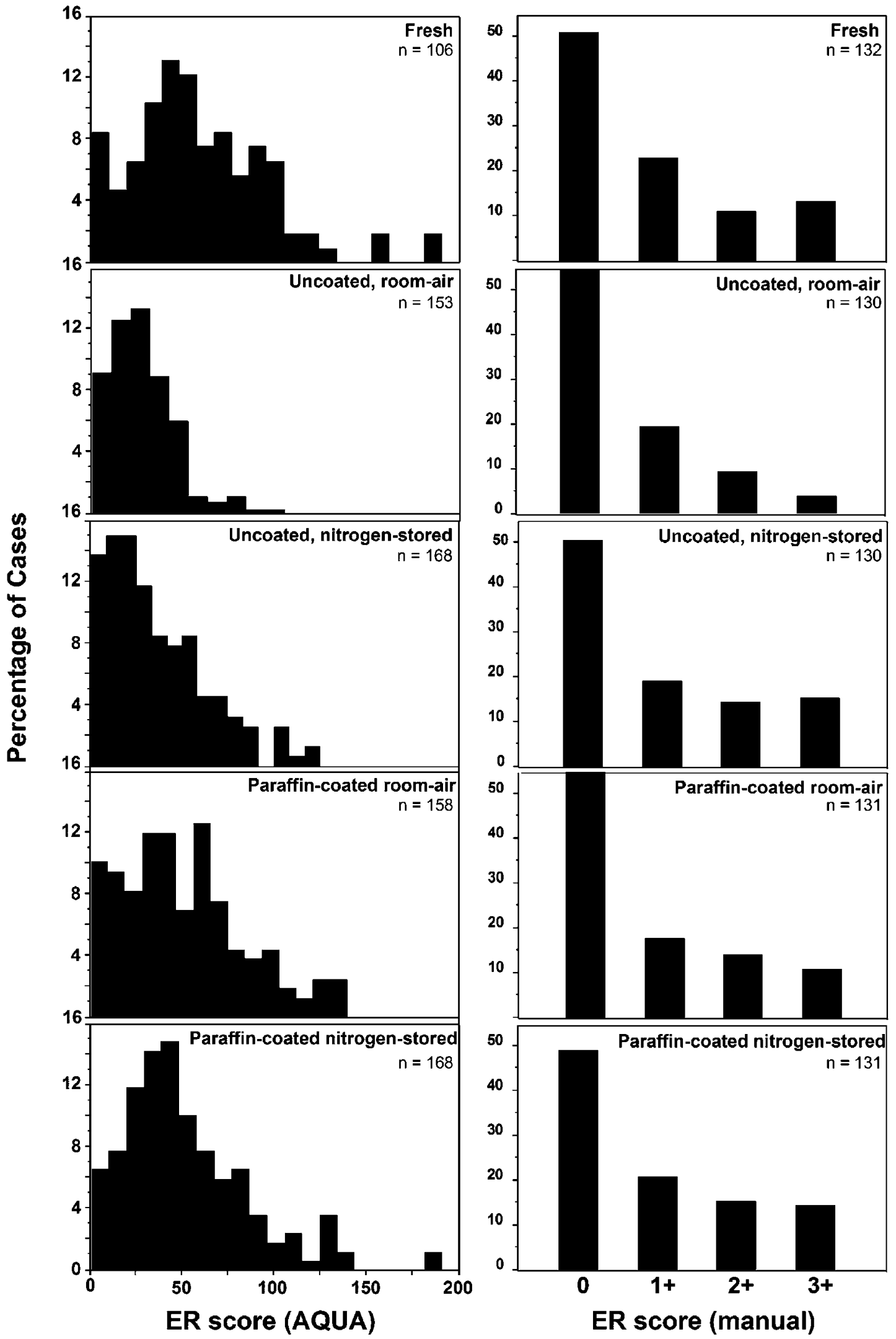

Figure 3 Paraffin coated and nitrogen storage of microarray slides retain ER antigenicity. Sections (5 $\mu$ m) of precut tissue microarrays were stored at room temperature in room air or in a nitrogen desiccation chamber. Some slides were coated with paraffin. After 3 months, the slides were immunohistochemically stained for ER and assayed using both manual and automated (AQUA) techniques. The percentage of cases at different ER levels are shown (0-3+ for the manual and 0-255 for automated scoring). 


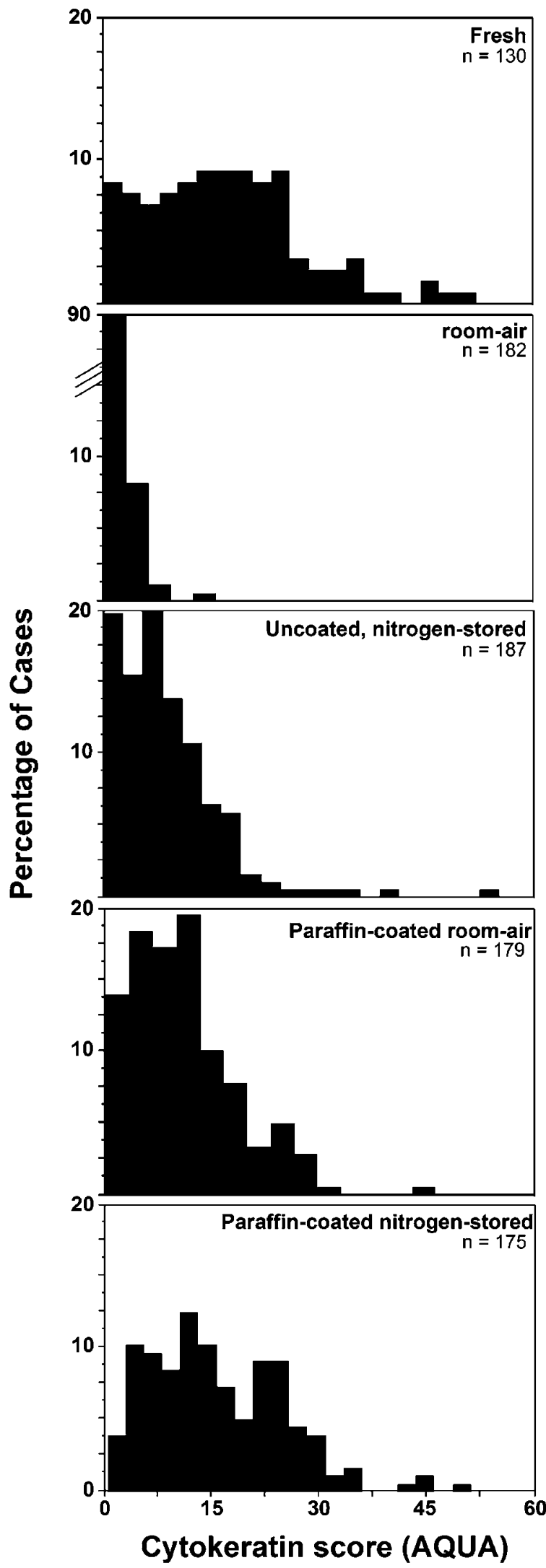

Table 3 Paired T-test of mean cytokeratin scores of stored slides stored for 3 months with nitrogen desiccation and/or paraffin coating

\begin{tabular}{lcr}
\hline Condition & \multicolumn{2}{c}{ Automated score } \\
\cline { 2 - 3 } & $\%$ Fresh & P-value \\
\hline Fresh & 100 & \\
Uncoated, room-air & 12 & $<0.0001$ \\
Uncoated, nitrogen & 54 & $<0.0001$ \\
Paraffin coated, room-air & 69 & $<0.0001$ \\
Paraffin coated, nitrogen & 96 & 0.4698
\end{tabular}

Table 4 Paired T-test of mean Ki-67 scores of stored slides stored for 3 months with nitrogen desiccation and/or paraffin coating

\begin{tabular}{lcc}
\hline Condition & \multicolumn{2}{c}{ Manual score } \\
\cline { 2 - 3 } & $\%$ Fresh & P-value \\
\hline Fresh & 100 & \\
Uncoated, room-air & 48 & $<0.0001$ \\
Uncoated, nitrogen & 59 & $<0.0001$ \\
Paraffin coated, room-air & 48 & $<0.0001$ \\
Paraffin coated, nitrogen & 72 & $<0.0001$ \\
\hline
\end{tabular}

Table 5 Paired $T$-test of mean ER scores of stored slides stored for 5 months under nitrogen, coated with paraffin containing various antioxidants

\begin{tabular}{lrc}
\hline Condition & \multicolumn{2}{c}{ Automated score } \\
\cline { 2 - 3 } & $\%$ Fresh & P-value \\
\hline Fresh & 100 & \\
No additive & 80 & $<0.0001$ \\
$1 \%$ BHT & 62 & $<0.0001$ \\
$1 \%$ BHA & 67 & $<0.0001$ \\
$10 \%$ BHT & 69 & $<0.0001$ \\
$10 \%$ BHA & 68 & $<0.0001$
\end{tabular}

In summary, tissue microarray slides stored under ambient conditions rapidly lose antigenicity; however, the magnitude of this loss differs from antigen to antigen. Although not completely protective, combined nitrogen storage and paraffin coating is a useful technique for preserving antigenicity for a period of up to 3 months. This technique prolongs the window of opportunity for staining microarray slides precut in batches.

Figure 4 Cytokeratin antigenicity is retained for 3 months on paraffin coated slides stored under nitrogen. Precut microarray slides, either paraffin-coated or -uncoated, were stored under ambient conditions or in a nitrogen desiccation chamber. After 3 months, the slides were immunohistochemically stained for cytokeratin and assayed using automated (AQUA) techniques. The percentages of cases exhibiting different cytokeratin staining levels are shown. 


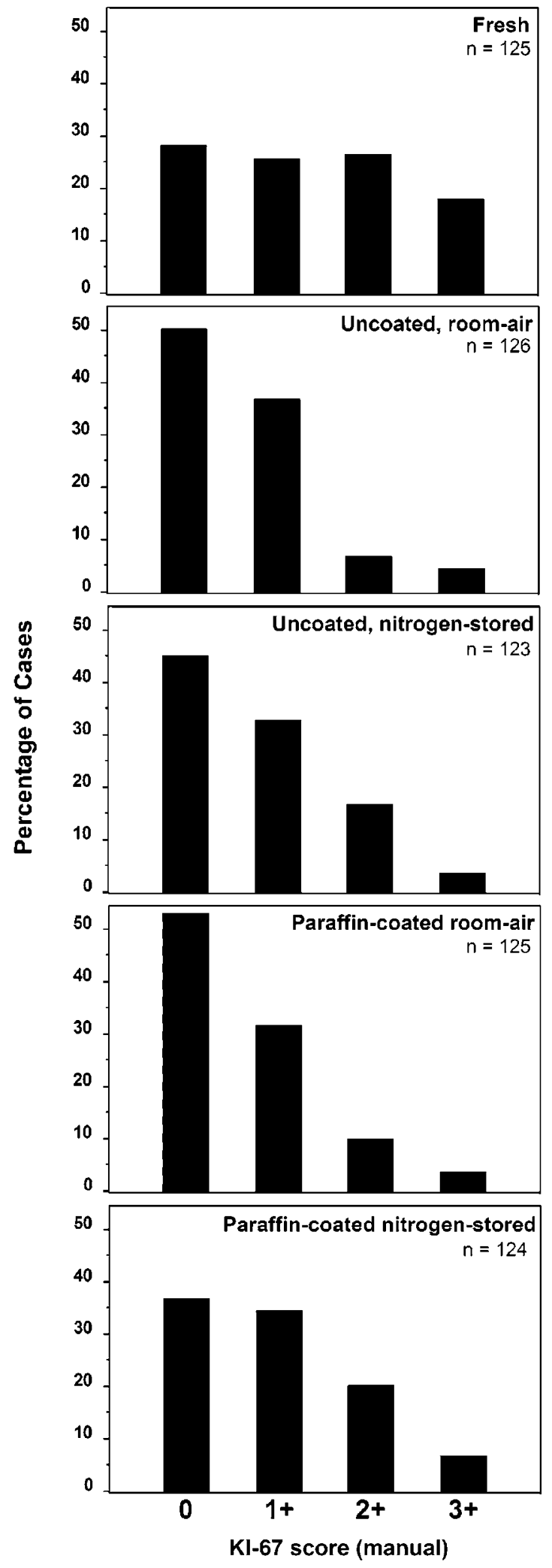

Figure 5 Ki-67 expression levels are partly retained by combined paraffin coating and nitrogen storage. Precut microarray slides were stored under various conditions for 3 months, stained for Ki-67, and scored manually. The percentage of cases exhibiting various levels of staining $(0-3+)$ are recorded.

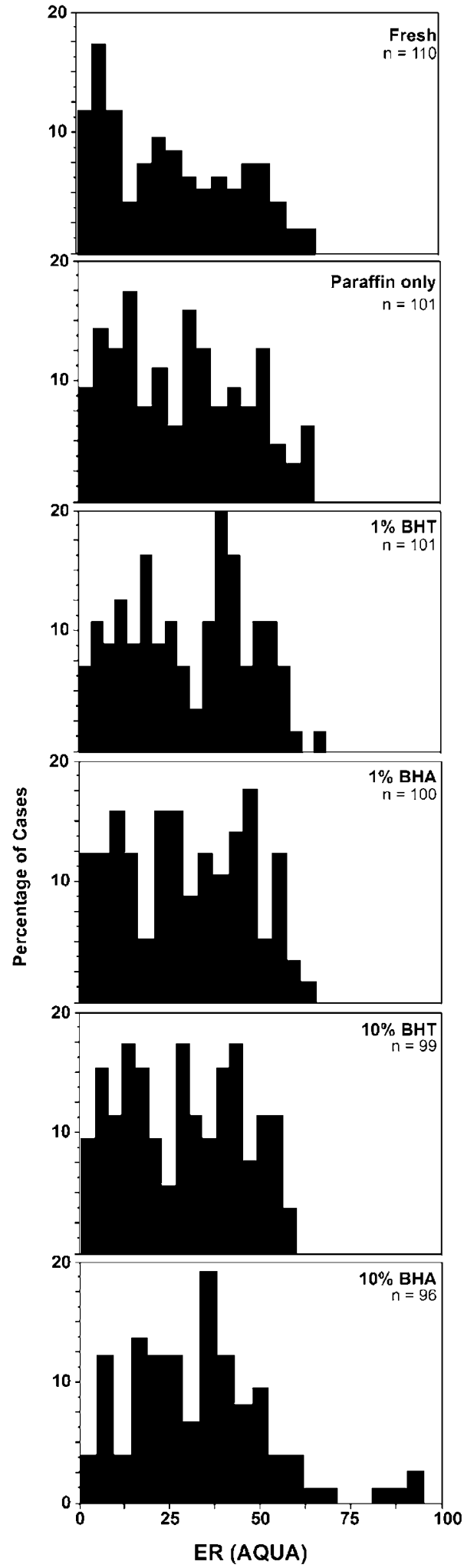

Figure 6 Addition of antioxidant additives to paraffin does not improve antigenicity. Sections $(5 \mu \mathrm{m})$ of pre-cut tissue microarrays were stored at room temperature in a nitrogen desiccation chamber for 5 months. Slides were either coated with paraffin with no additive, or with $1 \%(\mathrm{w} / \mathrm{v})$ or $10 \%(\mathrm{w} / \mathrm{v})$ BHA or BHT. The percentage of cases exhibiting various levels of staining by automated analysis are recorded. 


\section{Acknowledgements}

Dr RL Camp is supported by NIH Grant K0-8 ES11571 and by a grant from the Breast Cancer Alliance. Dr DL Rimm is supported by a grant from the Patrick and Catherine Weldon Donaghue Foundation for Medical Research, NIH Grant NCI R21 CA100825, and United States Army Grant DAMD17-02-0463.

\section{References}

1 Camp RL, Charette LA, Rimm DL. Validation of tissue microarray technology in breast carcinoma. Lab Invest 2000;80:1943-1949.

2 Manne U, Myers RB, Srivastava S, et al. Re: loss of tumor marker-immunostaining intensity on stored paraffin slides of breast cancer. J Natl Cancer Inst 1997; 89:585-586.

3 Bertheau P, Cazals-Hatem D, Meignin V, et al. Variability of immunohistochemical reactivity on stored paraffin slides. J Clin Pathol 1998;51:370-374.

4 Henson DE. Loss of p53-immunostaining intensity in breast cancer. J Natl Cancer Inst 1996;88:1015-1016.

5 Jacobs TW, Prioleau JE, Stillman IE, et al. Loss of tumor marker-immunostaining intensity on stored paraffin slides of breast cancer. J Natl Cancer Inst 1996;88: 1054-1059.

6 van den Broek LJ, van de Vijver MJ. Assessment of problems in diagnostic and research immunohisto- chemistry associated with epitope instability in stored paraffin sections. Appl Immunohistochem Mol Morphol 2000;8:316-321.

7 Fergenbaum JH, Garcia-Closas M, Hewitt SM, et al. Loss of antigenicity in stored sections of breast cancer tissue microarrays. Cancer Epidemiol Biomarkers Prev 2004;13:667-672.

8 Camp RL, Chung GG, Rimm DL. Automated subcellular localization and quantification of protein expression in tissue microarrays. Nat Med 2002;8:1323-1327.

9 Sen Gupta R, Hillemann D, Kubica T, et al. HOPEfixation enables improved PCR-based detection and differentiation of Mycobacterium tuberculosis complex in paraffin-embedded tissues. Pathol Res Pract 2003; 199:619-623.

10 Wester K, Asplund A, Backvall H, et al. Zinc-based fixative improves preservation of genomic DNA and proteins in histoprocessing of human tissues. Lab Invest 2003;83:889-899.

11 Beckstead JH. A simple technique for preservation of fixation-sensitive antigens in paraffin-embedded tissues. J Histochem Cytochem 1994;42:1127-1134.

12 Gillespie JW, Best CJ, Bichsel VE, et al. Evaluation of non-formalin tissue fixation for molecular profiling studies. Am J Pathol 2002;160:449-457.

13 Ahram M, Flaig MJ, Gillespie JW, et al. Evaluation of ethanol-fixed, paraffin-embedded tissues for proteomic applications. Proteomics 2003;3:413-421.

14 Vincek V, Nassiri M, Nadji M, et al. A tissue fixative that protects macromolecules (DNA, RNA, and protein) and histomorphology in clinical samples. Lab Invest 2003;83:1427-1435. 